\title{
Subchondral bone microstructural damage by increased remodelling aggravates experimental osteoarthritis preceded by osteoporosis
}

\author{
Miriam Bellido', Laura Lugo', Jorge A Roman-Blas', Santos Castañeda ${ }^{1,2}$, Jose R Caeiro ${ }^{3}$, Sonia Dapia ${ }^{3}$, \\ Emilio Calvo ${ }^{4}$, Raquel Largo ${ }^{1}$, Gabriel Herrero-Beaumont ${ }^{1 *}$
}

\begin{abstract}
Introduction: Osteoporosis (OP) increases cartilage damage in a combined rabbit model of OP and osteoarthritis $(\mathrm{OA})$. Accordingly, we assessed whether microstructure impairment at subchondral bone aggravates cartilage damage in this experimental model.

Methods: OP was induced in 20 female rabbits, by ovariectomy and intramuscular injections of methylprednisolone hemisuccinate for four weeks. Ten healthy animals were used as controls. At week 7, OA was surgically induced in left knees of all rabbits. At 22 weeks, after sacrifice, microstructure parameters were assessed by micro-computed tomography, and osteoprotegerin $(\mathrm{OPG})$, receptor activator of nuclear factor- $\kappa$ B ligand (RANKL), alkaline phosphatase (ALP) and metalloproteinase 9 (MMP9) protein expressions were evaluated by Western Blot at subchondral bone. In addition, cartilage damage was estimated using the histopathological Mankin score. Mann-Whitney and Spearman statistical tests were performed as appropriate, using SPSS software $\vee$ 11.0. Significant difference was established at $P<0.05$.
\end{abstract}

Results: Subchondral bone area/tissue area, trabecular thickness and polar moment of inertia were diminished in OPOA knees compared with control or OA knees $(P<0.05)$. A decrease of plate thickness, ALP expression and $\mathrm{OPG} / \mathrm{RANKL}$ ratio as well as an increased fractal dimension and MMP9 expression occurred at subchondral bone of $\mathrm{OA}$, OP and OPOA knees vs. controls $(P<0.05)$. In addition, the severity of cartilage damage was increased in OPOA knees vs. controls $(P<0.05)$. Remarkably, good correlations were observed between structural and remodelling parameters at subchondral bone, and furthermore, between subchondral structural parameters and cartilage Mankin score.

Conclusions: Microstructure impairment at subchondral bone associated with an increased remodelling aggravated cartilage damage in OA rabbits with previous OP. Our results suggest that an increased subchondral bone resorption may account for the exacerbation of cartilage damage when early OA and OP coexist simultaneously in same individuals.

\section{Introduction}

Osteoarthritis $(\mathrm{OA})$ is a multidimensional disease that affects all anatomical joint structures, particularly cartilage and subchondral bone [1,2]. In turn, osteoporosis (OP) is a skeletal disorder characterized by a compromised bone strength which substantially increases the

\footnotetext{
* Correspondence: gherrero@fjd.es

'Bone and Joint Research Unit, Service of Rheumatology. Fundación Jiménez Díaz, Universidad Autónoma. Avda. Reyes Católicos, 2. 28040 Madrid, Spain Full list of author information is available at the end of the article
}

risk of fracture [3]. Although OP and OA are two of the most prevalent skeletal disorders, both diseases are not frequently present in the same patient suggesting a mutually exclusive relationship. However, clinical studies often provide contradictory results which do not contribute to define the nature of the relationship between these medical conditions [4,5]. Furthermore, some confounding variables such as race, overweight and physical activity could explain this inverse relationship per se $[6,7]$. In addition, several studies may have been biased
C Biomed Central

C 2010 Bellido et al; licensee BioMed Central Ltd This is an open access article distributed under the terms of the Creative Commons Attribution License (http://creativecommons.org/licenses/by/2.0), which permits unrestricted use, distribution, and reproduction in any medium, provided the original work is properly cited 
by design deficiencies, no radiological confirmation for OA diagnosis, bad patient positioning at X-ray assessment and/or the presence of osteophytes [8,9]. In this context, animal models experiencing both pathologies without interference from other factors have become valuable tools. Indeed, in combined models of low bone mass and OA by destabilization, ovariectomy alone or associated with glucocorticoid administration increased joint damage $[10,11]$.

OA has been mostly considered a cartilage disorder. Nevertheless, the integrity of articular cartilage has been proposed to depend on mechanical properties of the underlying bone [12-14]. Several studies have recognized specific changes in the architecture and turnover of $\mathrm{OA}$ subcondral bone [15-17]. In fact, cartilage damage is frequently associated with thickening of the subchondral plate and osteophytosis during knee OA in humans [14-16,18] and animals [19]. But besides this hypertrophic OA, some authors contemplate another variant, the atrophic form, which is characterized by the lack of osteophytes and loss of subchondral bone volume in OA patients with hip and knee compromise [20-23]. Furthermore, the correlation between the serum levels of both C-propeptide and collagenase of type II collagen observed in hypertrophic OA was lost in atrophic OA, where exists a reduced type II collagen synthesis [24]. This could contribute to the absence of osteophyte formation, as well as to the increased subchondral bone turnover in rapidly progressive hip and knee OA $[25,26]$. Remarkably, the presence of subchondral bone attrition in knee OA, defined as flattening or depression of the osseous articular surface, is strongly associated with subchondral bone marrow lesions (BMLs) on MRI, In turn, BMLs reflect the presence of active remodeling processes due to chronic overload [23]. Cartilage loss occurred in the same knee subregions as subchondral bone attrition [27]. Likewise, subchondral bone in OA animal models with early stages of the disease have shown both decreased volume and stiffness $[28,29]$, and increased remodelling [29]. The impact of these subchondral bone changes in OA is still debated, partially due to heterogeneity of the disease [30].

Different molecular alterations have been described to take place in the remodelling of OA subchondral bone [31]. In this regard, the receptor activator of nuclear factor $-\kappa \mathrm{B}$ ligand (RANKL) and the osteoprotegerin (OPG), members of the RANKL/RANK/OPG pathway, are expressed differently whether the subchondral bone is affected or not by OA $[32,33]$. This pathway has been acknowledged as a key mechanism in the regulation of osteoclast formation or activation [34]. Although subchondral bone impairment seems to play a relevant role in $\mathrm{OA}$ onset and progression [31], the underlying mechanisms remain unknown. Therefore, an improved understanding of these mechanisms would increase our knowledge in the contribution of subchondral bone on cartilage damage and on its suitability as a therapeutic target in OA.

Thus, the present study aimed to assess whether microstructure impairment at subchondral bone may enhance cartilage damage when OA and OP are present simultaneously in rabbits. Furthermore, we characterize several bone microstructural parameters and metabolic modulators involved in subchondral remodelling as well as their relationships in these rabbits with OA aggravated by previous OP.

\section{Materials and methods \\ Animals}

Thirty skeletally mature female, eight-month-old (3.8 to $4.8 \mathrm{~kg}$ body weight), New Zealand rabbits were included in the study (Granja Universal, Pamplona, Spain). The animals became acclimatized after two weeks and were housed individually in stainless-steel cages and maintained on a 12-h light/12-h dark cycle at room temperature. The animals had free access to water and standard rabbit chow (Panlab, Barcelona, Spain). The guidelines for care and use of animals were followed throughout the study, in accordance with procedures approved by the Institutional Review Board.

\section{Experimental animal model}

OP was induced in 20 rabbits, by ovariectomy and intramuscular injections of methylprednisolone hemisuccinate ( $1 \mathrm{mg} / \mathrm{kg} /$ day for four weeks; OP group). Ten age- and gender-matched additional animals were used as controls (healthy group). Surgical OA was induced in the left knees in all rabbits through medial meniscectomy and anterior cruciate ligament (ACL) section seven weeks after the beginning of the study. For ovariectomy and experimental OA, all rabbits were anesthetized and antibiotic prophylaxis was used according to protocol previously described [11]. Left knees were considered as OA $(n=10)$ or as OA and OP (OPOA; $n=20)$ and right knees were used as OP $(n=20)$ or healthy controls $(n=10)$, respectively. All animals were permitted free cage activity after surgery. Rabbits were euthanized by intracardiac administration of sodium pentobarbital (50 mg/kg; Pentotal, Abbott, Madrid, Spain) 15 weeks after knee surgery (Figure 1A). At sacrifice, samples of articular cartilage and subchondral bone of each knee were collected for further histological and Western Blot analysis or microstructural studies. Serum samples were taken for determining total alkaline phosphatase (ALP) and tartrate-resistant alkaline phosphastase (TRAP) activity assays at baseline and at $6,12,16$ and 21 weeks after the study onset. All the experiments were approved by the local ethics committee. 


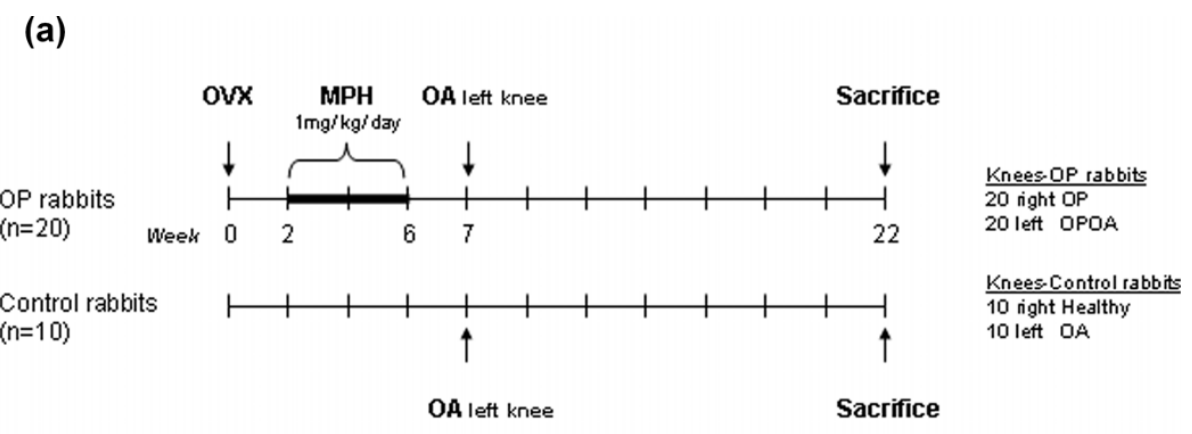

(b)

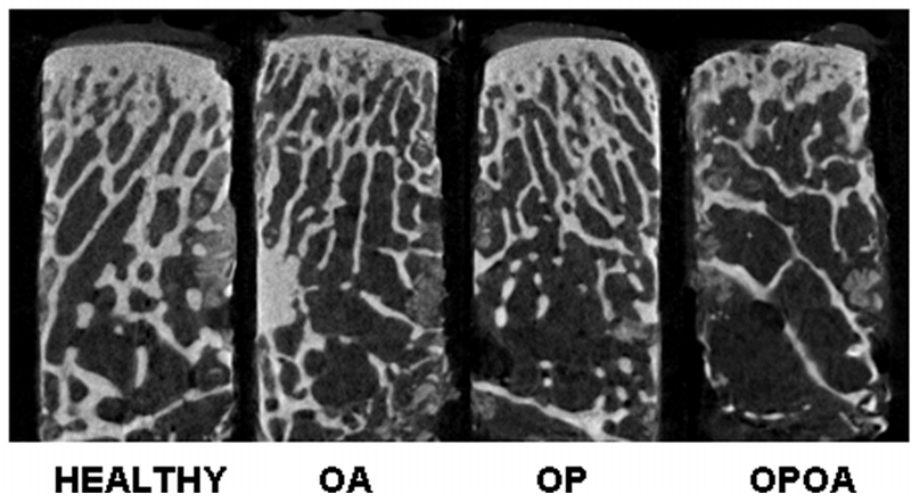

Figure 1 Experimental design. This study evaluated the effect of increased remodelling on subchondral bone microstructure and biomechanical-related genometrics in a combined rabbit model of osteoarthritis (OA) aggravated by previous osteoporosis (OP). (a)Experimental scheme. OVX: ovariectomy; MPH: methylprednisolone hemysuccinate. (b)Qualitative 2D models were reconstructed from images selected of a highly representative sample for each group (HEALTHY, OA, OP and OPOA), by using the CTAn software (Skyscan, Aartselaar, Belgium).

\section{Subchondral bone microstructural study}

Cylindrical biopsies of subchondral bone with the size of $9 \mathrm{~mm}$ in length by $4 \mathrm{~mm}$ in diameter were extracted from femoral condyles with a stainless steel trepan bur for explantation (Group Komet, Lemgo, Germany). The trepan bur was connected to a surgical motor (KaVo Dental GmbH, Biberach, Germany) that provided a speed of 1,500 rpm under continuous irrigation with saline soultion. Following this, the obtained biopsies were hydrated in saline solution and frozen at $-20^{\circ} \mathrm{C}$ for subsequent analysis.

The microarchitecture of the samples was studied using micro-computed tomography ( $\mu$ CT; Skyscan 1172; Skyscan, Aartselaar, Belgium). The X-ray source was set at $100 \mathrm{kV}$ and $100 \mu \mathrm{A}$, with a pixel size of $10.9 \mu \mathrm{m}$. Four hundred and fifty projections were acquired over an angular range of $180^{\circ}$ (angular step of $0.40^{\circ}$ ). Image slices were reconstructed using the NRecon software (Skyscan, Aartselaar, Belgium) based on the Feldkamp algorithm, and applying corrections for beam hardening and ring artifacts. Registered datasets were segmented into binary images using global thresholding methods. The trabecular bone was extracted by drawing round contours with CTAn software (Skyscan, Aartselaar,
Belgium) and the regions of interest selected were analyzed with the same application (see Figure 1B).

The quantitative structural two-dimensional (2D) variables determined for selected regions were: tissue area (T.Ar), bone area (B.Ar), bone area fraction (B.Ar/T.Ar $\%)$, trabecular thickness ( $\mathrm{Tb} . \mathrm{Th}$ ), trabecular number ( $\mathrm{Tb}$. $\mathrm{N})$ and trabecular separation (Tb.Sp). Furthermore, we assessed the thickness of the first layer of subchondral plate by measuring the distance between the subchondral bone edge and the point where the value of B.Ar/T. Ar drops to more than $98 \%$ of its value at the bone edge. This point would indicate the presence of subchondral trabecular bone. In addition, the fractal dimension (FD) and polar moment of inertia (Ip) were calculated for each sample. FD was measured as complexity index of the bone microarchitecture [35], and calculated using the Kolmogorov or box counting method by the Skyscan CTAn software. For 2D calculation of FD, the cross-sectional image was divided into an array of equal squares, and the number of squares containing part of the bone surface is counted. This was repeated over a range of square sizes such as 2 to 100 pixels. The number of squares containing the bone surface is plotted against square length in a log-log plot, 
and finally FD was obtained from the slope of the loglog regression. In turn, Ip was assessed as geometric index of bone strength to resist torsion [36]. Furthermore, the moment of inertia represents the rotational analogue of mass for linear motion. For a point mass (represented by an image pixel) the moment of inertia (I) is the mass $(\mathrm{m})$ times the square of perpendicular distance $(r)$ to the rotation axis, $\mathrm{I}=\mathrm{mr}^{2}$.

\section{Western Blot analysis}

For OPG, RANKL, ALP and MMP9 protein expression studies, $40 \mu \mathrm{g}$ of total protein from tibial subchondral bone were loaded and then resolved on $10 \%$ acrylamideSDS gels. After transfer to polyvinylidene difluoride (PVDF) membranes (Millipore, Molsheim, France), membranes were blocked in 5\% skimmed milk in PBSTween 20 for $1 \mathrm{~h}$ at room temperature, and incubated overnight at $4^{\circ} \mathrm{C}$ with primary antibodies. These antibodies included goat anti-OPG (R\&D Systems, Abingdon, UK) at $1 / 1000$ dilution, rabbit anti-sRANKL (Prepotech, Neuilly-Sur-Seine, France) at 1/500, mouse anti-ALP (Abcam, Cambridge, UK) at 1/500, and mouse antiMMP9 (Calbiochem/Merck Chemicals, Nottingham, UK) at $1 / 500$. Later, antibody binding was detected by enhanced chemoluminiscence using peroxidase-labelled secondary antibodies and densitometric results were expressed in arbitrary units (AU) normalized to Glyceraldehyde 3-phosphate dehydrogenase (GAPDH) levels.

\section{Serum total ALP and TRAP activity assay}

Serum ALP activity was determined by colorimetry, using p-nitrophenyl phosphate as substrate in a glycine buffer (glycine $50 \mathrm{mM} ; \mathrm{Cl}_{2} \mathrm{Mg} 0.5 \mathrm{mM}, \mathrm{pH}$ 10.5). Serum samples were incubated at $37^{\circ} \mathrm{C}$ during 60 minutes, and then the hydrolysis reaction was stopped with $\mathrm{NaOH}$ $0.02 \mathrm{~N}$. Finally, absorbance was measured at $405 \mathrm{~nm}$, since the serum ALP activity is directly proportional to the amount of substrate degraded. For the TRAP kinetic assay, the method was similar but the samples were incubated in a sodium acetate $50 \mathrm{mM}$ and sodium tartrate $10 \mathrm{mM}$ ( $\mathrm{pH} 4.8$ ) buffer and the reaction was stopped with $\mathrm{NaOH} 0.1 \mathrm{~N}$.

\section{Cartilage histology}

After sacrifice, femur and tibia sections were fixed in buffered formalin for $24 \mathrm{~h}$ and then decalcified for six weeks in an ethylenediaminetetraacetic acid (EDTA) solution (2 mM EDTA, $0.5 \mathrm{mM}$ tartrate sodium potassium, pH 1) for further histological evaluation.

The decalcified knee joints were cleaved in a sagital plane along the central portion of the articular surface of each medial femoral condyle corresponding to the weightbearing area, before embedding in paraffin wax.
Cartilage sections $(5 \mu \mathrm{m})$ were stained with hematoxylin and eosin to assess cellularity and structural abnormalities, and with Alcian blue to evaluate matrix abnormalities. The weight-bearing area of the femoral condyle was delimited and histopathologically assessed using the Mankin's grading system [37] by an experienced cartilage pathologist. The observer was blinded with respect to the rabbit group, laterality and macroscopic description. Samples were presented to the observer in random order. A partial score for each scale category (structure abnormalities, cellularity, matrix staining and tidemark integrity) was allocated, and the scores in each of these categories were combined for every section. The evaluation was performed at the weight bearing surface of the medial femoral condyle because it shows the earliest and most severe histological abnormalities [11,38].

\section{Statistical analysis}

Results are expressed as mean \pm standard error of the mean (SEM). Data from multiple groups were compared using Mann-Whitney non-parametric analyses as appropriate. Correlations were evaluated using the Spearman test. All statistical analyses were performed using commercially available software (SPSS v 11.0, SPSS Inc, Chicago, USA). Differences were considered significant when $P<0.05$.

\section{Results}

\section{Microstructure impairment at subchondral bone}

Changes in subchondral microstructure were studied 15 weeks after meniscectomy and ACL section of the knee to induce OA in a rabbit OP model (Figure 2A). Indeed, healthy knees showed a B.Ar/T.Ar higher than OPOA group $(P<0.05)$. Moreover, B.Ar/T.Ar in OPOA knees was decreased with respect to that in OA knees $(P<$ 0.05). Similarly, Tb.Th in groups affected by OP or both conditions showed a downward trend with respect to healthy controls, however, only the combined OPOA group showed a significant decrease $(P<0.05)$. Conversely, Tb.Sp was increased in OP and OPOA knees with respect to healthy group (healthy: $0.24 \pm 0.01 \mathrm{~mm}$; OP: $0.28 \pm 0.02 \mathrm{~mm}$, and OPOA: $0.31 \pm 0.02 \mathrm{~mm}$ ), yet only OPOA significatively increased it $(P<0.05)$. Regarding Tb.N, no differences were found between the healthy and other groups $(P>0.05)$.

Notably, the plate thickness of subchondral bone was diminished in OA, OP or OPOA groups when compared with the healthy one $(P<0.05)$. Even more, the combined OPOA displayed a more decreased subchondral plate thickness than the OA group $(P=0.004)$, see Figure 2B. In addition, the microarchitecture index FD was increased in all groups $v s$. healthy $(P<0.05)$, and notably the OPOA group presented the highest difference against 
(a)
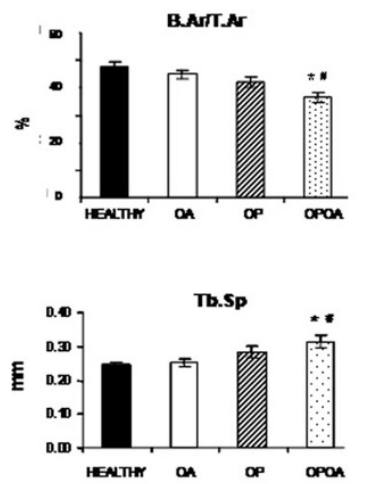
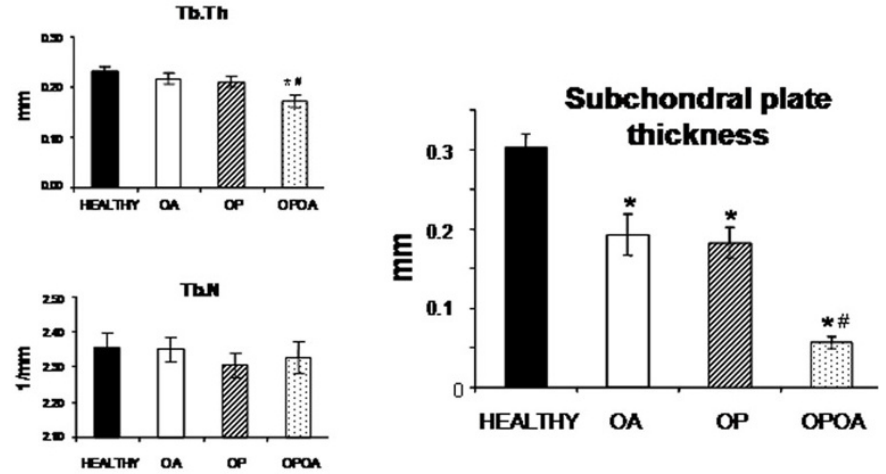

(c)
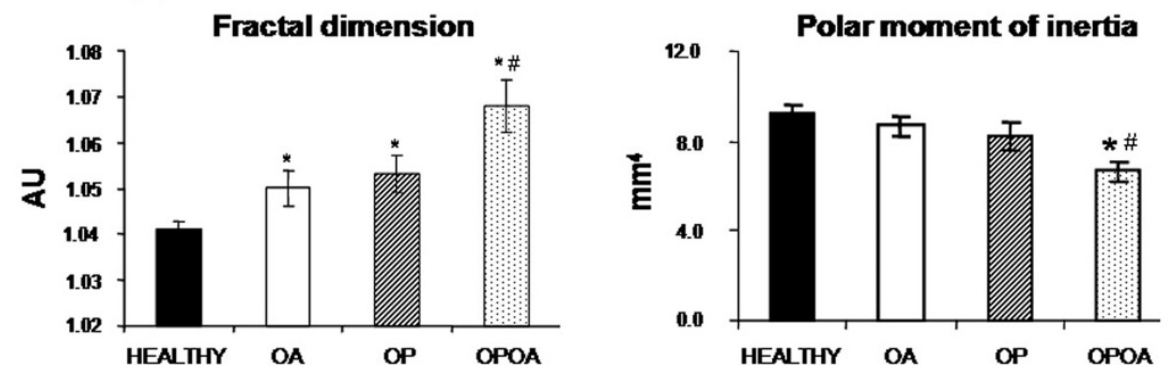

Figure 2 Microstructure parameters at subchondral bone. (a)Bone area fraction (B.Ar/T.Ar), trabecular thickness (Tb.Th), trabecular number (Tb.N) and trabecular separation (Tb.Sp) were assessed at subchondral bone. (b)Subchondral plate thickness. (c)Biomechanical-related structural parameters, fractal dimension (FD) and polar moment of inertia (Ip), at subchondral bone. HEALTHY $(n=7), \mathrm{OA}(n=7), \mathrm{OP}(n=8)$ and OPOA $(n$ = 8) knees. OA, osteoarthritis; OP, osteoporosis. Values are expressed as mean $\pm \mathrm{SEM}$; ${ }^{*} P<0.05$ vs. HEALTHY, \#P 0.05 vs. OA.

healthy or OA groups (Figure 2C). Furthermore, the biomechanical-related index Ip tended to be lower in both OA and OP groups than healthy knees, but they did not reach a statistical significance. However, Ip value of OPOA group was decreased when compared to healthy or OA groups $(P<0.05)$ (Figure $2 \mathrm{C}$ ).

\section{ALP and MMP9 protein expression at subchondral bone}

Remodelling was estimated in each group by measuring the changes in protein expression of ALP and MMP9 at subchondral bone on week 22 (Figure 3A). Subchondral bone formation assessed by ALP protein expression was diminished in OA, OP and OPOA groups vs. healthy knees (healthy: $100 \pm 3$ AU; OA: $90 \pm 1$ AU; OP: $90 \pm 2$ AU; and, OPOA: $75 \pm 2$ AU; $P<0.05$ in all cases). Furthermore, subchondral ALP expression in OPOA group also decreased with respect to that in OA group. In turn, subchondral bone degradation estimated by MMP9 protein expression was highly increased in OA, OP and OPOA groups with regard to healthy knees $(P$ $<0.01$ in all cases). However, although there was a tendency to increase, subchondral MMP9 expression in the combined group did not show a difference with respect to that in OA or OP groups.

\section{Increased serum ALP and TRAP enzymatic activities}

In addition, serum enzymatic activities of ALP and TRAP were assayed in order to study the status of systemic bone turnover in our experimental animals. This analysis showed a high remodelling process in OP rabbits with or without OA (OP and OPOA groups), compared with those non-OP rabbits (healthy and OA groups). Indeed, an increase in both ALP and TRAP activity was found in OP and OPOA groups at week 6 (Figure 3B). Serum ALP activity was transiently increased until week 12 and restored to normal levels afterward in all groups. However, serum TRAP remained high throughout the study in OP rabbits with or without OA. It should be noted that systemic enzymatic activity measurements in OP or OPOA groups were the same since their knees come from same OP animals whether or not they underwent surgical OA. A similar situation occurred for healthy or OA groups that come from same non-exposed animals to OP stimulus.

\section{OPG/RANKL ratio at subchondral bone}

To determine the changes in RANKL/RANK/OPG pathway that occur in OA aggravated by previous OP, we have compared subchondral bone OPG and RANKL protein 
(a)

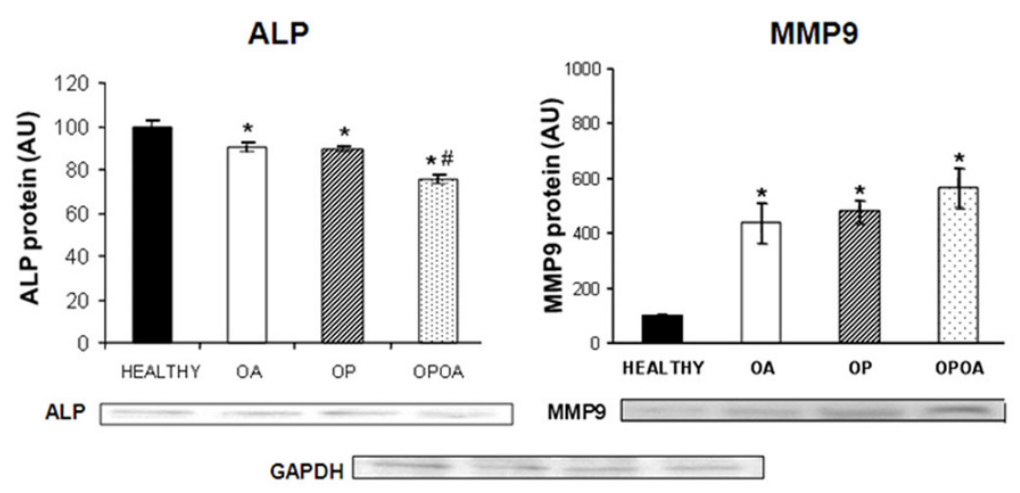

(b)

ALP

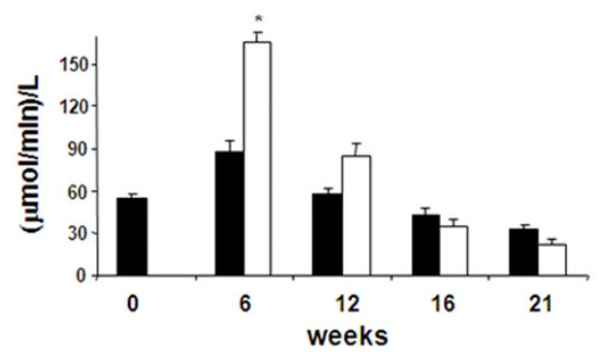

TRAP

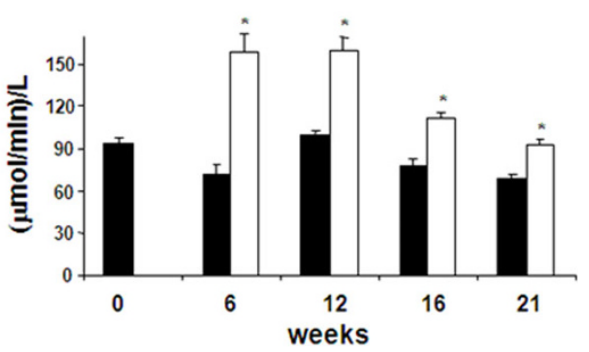

Figure 3 Remodelling parameters at subchondral bone and systemic level. (a)Alkaline hosphatise (ALP) and metalloproteinase 9 (MMP9) protein expressions at subchondral bone. At top: Densitometric analysis of ALP and MMP9 protein expressions at subchondral bone. At bottom: representative Western Blot images of ALP and MMP9 for $40 \mu \mathrm{g}$ of total subchondral bone protein. The number of samples assayed for ALP expression were: HEALTHY $n=10$; OA $n=8$; OP $n=8$ and OPOA $n=10$, and for MMP9 were: $n=6$ in each group. OA, osteoarthritis; OP, osteoporosis (b)Enzymatic activity for ALP and tartrato-resistant alkaline phosphatase (TRAP) in serum from OP animals (OP and OPOA groups; $n$ $=8$; white bars) and non-OP animals (healthy and OA groups; $n=7$; black bars). OA, osteoarthritis; OP, osteoporosis. Bar represents the mean \pm SEM. ${ }^{*} P<0.05$ vs. HEALTHY, \#P $<0.05$ vs. OA.

expression between groups by Western Blot (Figure 4). OPG synthesis was decreased in all experimental groups respect to healthy knees (healthy: $100 \pm 6 \mathrm{AU}$; OA: $78 \pm 6$ AU; OP: $63 \pm 5 \mathrm{AU}$, and OPOA: $69 \pm 3 \mathrm{AU} ; P<0.05$ in all cases). RANKL expression was only increased in the OPOA group vs. healthy or OP groups (healthy: $100 \pm 11$ AU; OA:112 \pm 8 AU; OP:93 \pm 5 AU, and OPOA:135 \pm 9 AU; $P<0.05)$. In addition, we have assessed the OPG/ RANKL ratio finding a decrease in experimental groups when compared to healthy group. Furthermore, the combined group experienced a greater reduction in this ratio than OA group $(P<0.05)$.

\section{Cartilage histopathology}

The impact of previous OP upon cartilage integrity in OA knees was estimated by using the histopathological Mankin grading score, see Figure 5. Thus, OPOA knees showed higher total Mankin scores than control knees $(P<0.05)$. Moreover, there were also differences when the scores obtained from OPOA knees were compared with OA knees. Overall Mankin scores in OA knees were higher than those in OP knees, but the differences were not significant. However, OP knees showed higher scores than normal healthy knees $(P<0.05)$, suggesting that OP could have an aggravating detrimental effect in the development of OA lesions.

\section{Correlations between structural and remodelling} parameters at subchondral bone with cartilage damage The associations between remodelling and structural parameters or OPG/RANKL ratio at subchondral bone were assessed in the different groups. Spearman correlations analysis (Table 1) showed that B.Ar/T.Ar\% was directly associated with plate thickness and Ip at subchondral bone $(P<0.05)$, whereas, FD was inversely associated to the other structural parameters $(P<0.05)$. In the same way, at subchondral bone, the relationships were significant between ALP and the structural parameters B.Ar/T.Ar\%, Ip or FD as well as between MMP9 and OPG/RANKL ratio. Moreover, there were significant correlations between subchondral bone structural parameters or ALP expression and cartilage damage. 


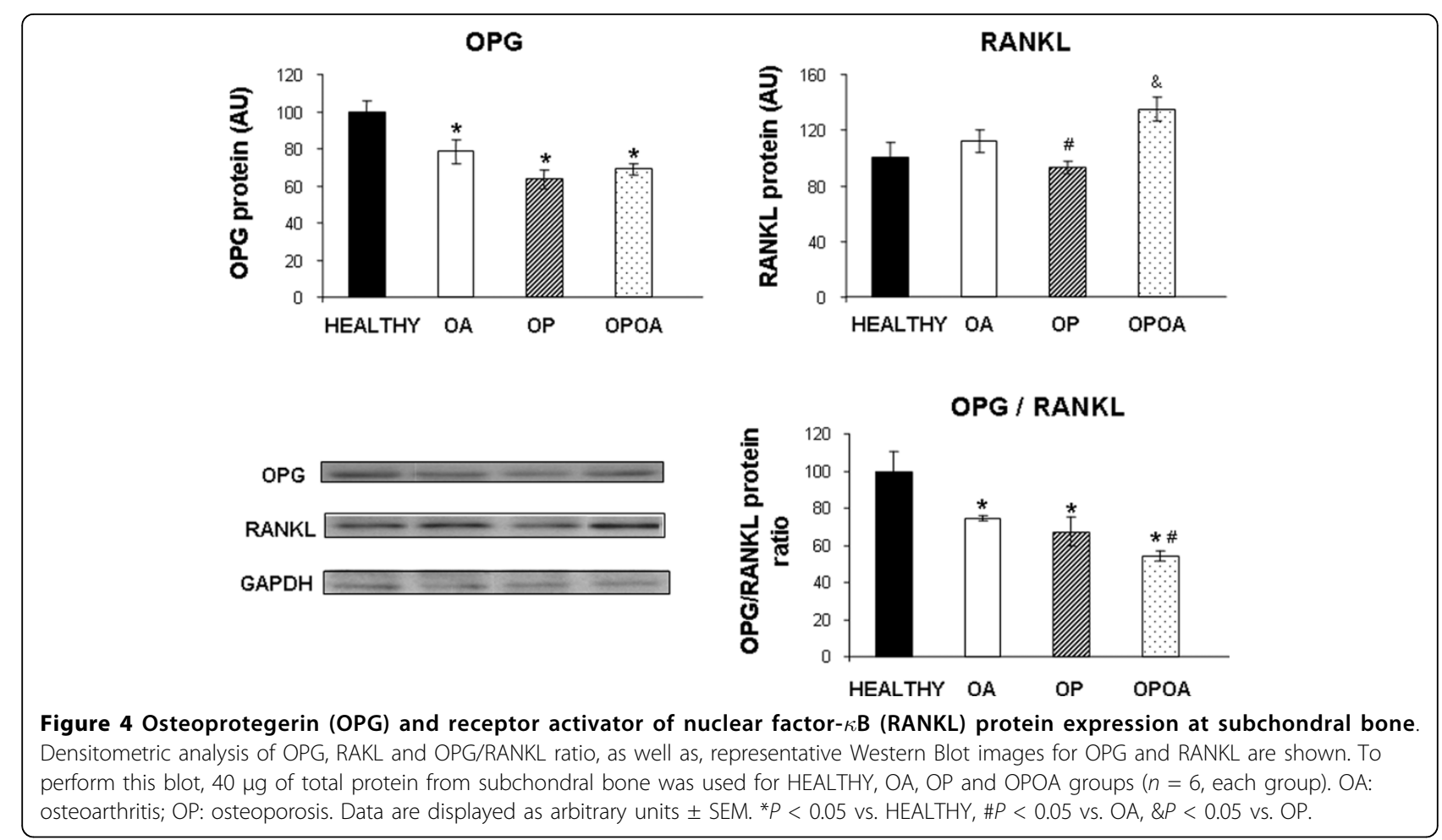

\section{Discussion}

We have studied the alterations in the microstructure of subchondral bone originated by an increased local bone resorption, as well as their relevant relationships with cartilage damage in the knees of OA rabbits with previous OP. Most of clinical studies suggest an inverse relationship between OP and OA. However, a negative OP effect upon articular cartilage integrity has been described in animal models [10,39-41]. Cartilage damage

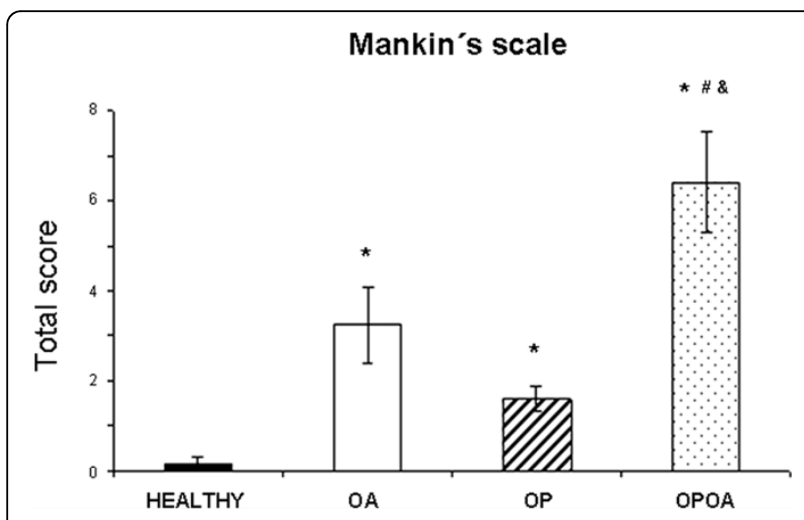

Figure 5 Total Mankin score of histological cartilage damage. The assessment was carried out at the weight bearing area of medial femoral condyle. HEALTHY $(n=7), \mathrm{OA}(n=7), \mathrm{OP}(n=8)$ and OPOA $(n=8)$ knees. OA: osteoarthritis; OP: osteoporosis. Results are expressed as mean \pm SEM; ${ }^{*} P<0.05$ vs. HEALTHY, $\# P<0.05$ vs. $\mathrm{OA}, \& P<0.05$ vs. OP. has been recognized not only by an impairment of structural and biomechanical properties, but also by identifying changes in matrix composition or collagen degradation in these OP models. In this sense, our group has reported that previous OP induces cartilage injury in a combined rabbit model of OP and OA [11].

In the current study, the knees presenting simultaneously OP and OA exhibited the thinnest subchondral plate, decreased B.Ar/TAr and $\mathrm{Tb} . \mathrm{Th}$, as well as increased Tb.Sp. In turn, the rabbits belonging to OP and OA groups showed a decrease in subchondral plate thickness and only a negative tendency in B.Ar/TAr and Tb.Th values. The decrease of subchondral plate thickness in OP, OA and particularly its pronounced decline in OPOA knees would indicate that our experimental model exhibits a much more profound effect in subchondral cortical bone than in subchondral trabecular bone. In agreement with our results, similar findings were observed in both murine and canine models of OA $[28,42,43]$. However, a thickening of subchondral plate was described in the guinea pig OA model by partial meniscectomy [19] and in the ACL transection-induced OA in rat model $[44,45]$. It is worth noting that subchondral microstructure changes in latter models corresponded to an advanced OA stage $[19,44,45]$. In addition, we have performed a microstructure analysis of the subchondral bone in a cylinder sample until 9 mm deep, thus studying not only the first cortical layer, but also the trabecular bone beneath. Consequently, our 
Table 1 Associations between structural, remodelling and molecular parameters at subchondral bone, and cartilage damage score

\begin{tabular}{|c|c|c|c|c|c|c|c|c|}
\hline & B.Ar/T.Ar\% & Sb. Th & FD & Ip & ALP & MMP9 & OPG/RANKL & MANKIN \\
\hline B.Ar/T.Ar\% & 1.000 & & & & & & & \\
\hline Sb. Th & $0.381^{*}$ & 1.000 & & & & & & \\
\hline FD & $-0.861^{* *}$ & $-0.450^{*}$ & 1.000 & & & & & \\
\hline Ip & $0.963^{* *}$ & 0.347 & $-0.868^{* *}$ & 1.000 & & & & \\
\hline ALP & $0.814^{* *}$ & 0.453 & $-0.754^{* *}$ & $0.718^{* *}$ & 1.000 & & & \\
\hline MMP9 & -0.115 & -0.195 & 0.262 & -0.094 & -0.311 & 1.000 & & \\
\hline OPG/RANKL & -0.280 & -0.120 & 0.340 & -0.247 & -0.054 & $0.500^{*}$ & 1.000 & \\
\hline MANKIN & $-0.627^{* *}$ & $-0.626^{* *}$ & $0.672^{* *}$ & $-0.598^{* *}$ & $-0.788^{* *}$ & 0.460 & 0.341 & 1.000 \\
\hline
\end{tabular}

* Correlation is significant at the 0.05 level (2-tailed).

** Correlation is significant at the 0.001 level (2-tailed).

The correlation coefficients represented in this table are obtained comparing the healthy, OA, OP and OPOA groups by Spearman bivariate analysis.

B.Ar/T.Ar\%, bone area fraction; FD, fractal dimension; Ip, polar moment of inertia; MANKIN, cartilage histopathological Mankin grading scorel; MMP9,

metalloproteinase 9; OA, osteoarthritis; OP, osteoporosis; OPG/RANKL ratio, osteoprotegerin/receptor activator of nuclear factor- $\kappa B$ ratio; Sb.Th, subchondral plate thickness.

differences in respect to other studies could be derived from both the stage of OA development and the subchondral region of analysis.

Bone is an anisotropic material since its mechanical properties vary in different directions. In this context, an estimated mechanical stiffness of the trabecular bone can be obtained by an indirect method that considers the combination of bone volume fraction and architectural anisotropy. This method, the FD analysis, assesses the directional anisotropy of trabecular bone [46]. Other studies have also revealed that 2D fractal analysis relates to three-dimensional parameters such as porosity and connectivity [35]. In this way, FD in the combined model was increased, suggesting that subchondral bone that supports the articular cartilage in OPOA knees is more porous than that in other groups. Using fractal analysis by macroradiographs, it has been described a localized OP in large human OA joints $[47,48]$. On the other hand, bone architectural efficiency to resist torsion loads can be evaluated by the Ip, which is a relevant biomechanical bone factor. Thus, the higher the Ip, the stronger and more resistant the bone is to bearing torsion loads. By contrast, lower Ip drives to less bone stiffness [49]. In our study, this factor was lower in the OPOA group respect to those in other groups, hence suggesting a higher fragility of the subchondral bone of OPOA knees.

Bone remodelling is a finely regulated process resulting in the coordinated resorption and formation of skeletal tissue. The increase of systemic ALP and TRAP levels at week 6 in OP animals with and without OA (OP and OPOA groups) confirmed the presence of a high remodelling process during early OP stages, immediately after ovariectomy and glucocorticoid administration. However, on later days, the formation/resorption balance was oriented toward the resorption as TRAP activity continued high in OP rabbits with or without OA. Our results are in agreement with previous studies that have shown increased subchondral ALP levels at 38 days after ovariectomy in rats [50]. Moreover, in our study, OP, OA, and OPOA groups presented a lower ALP expression at subchondral bone which reflects the systemic turnover changes seen at week 21 . In contrast, other authors reported the enzymatic ALP activity as invariable in OP or increased in OA [51]. These differences could be due to the late OA stage studied in human joints [51], while ALP assays in animal models have been performed at an early OA stage [29]. Furthermore, the negative glucocorticoid effects on bone formation in our experimental model could be reflected in the decrease of subchondral ALP synthesis in OPOA knees.

MMP9 expression was further analyzed as an index of the prominent enzymatic degradation of the extracellular matrix of subchondral bone and cartilage exerted by the MMPs. Furthermore, the presence of subchondral bone resorption pits composed by MMP-producing cells derived form bone marrow has been evidenced and they contribute to cartilage degradation [52]. Previous studies have described an increased MMP9 protein/gene expression not only in the subchondral bone but also in articular cartilage in both human and experimental OA studies $[45,53,54]$. Accordingly, we found raised subchondral MMP9 protein levels in OP, OA and OPOA groups demonstrating a clear increase in local resorption. Furthermore, subchondral MMP9 levels may reflect the increased serum TRAP activity seen in OP animals with or without OA (OP and OPOA groups) at week 21. In addition, to our knowledge, this study shows for the first time MMP9 up-regulation in an OP experimental model, which supports the role of this enzyme in bone resorption during OP. Taken together, 
all these findings indicate that OP increases OA subchondral remodelling through stimulation of bone resorption, and suggest that this detrimental effect may be particular relevant at early OA.

Remarkably, the changes at subchondral microstructure and remodelling were supported by the results obtained at the molecular level in this study. In fact, the subchondral OPG/RANKL ratio was decreased in the OA, OP and OPOA groups when compared to controls, suggesting a clear increase in subchondral bone resorption in all these conditions. Moreover, OPOA showed a smaller OPG/RANKL ratio than OP or OA alone, therefore, indicating a greater subchondral bone resorption in the combined group. These results are in line with previous studies in OP and OA. RANKL is the principal regulator of bone resorption by promoting $\mathrm{OC}$ differentiation, activity, and survival. OPG is a decoy receptor that binds to RANKL inhibiting $\mathrm{OC}$ formation and survival [55]. A decrease in the OPG/RANKL ratio is involved in the development of post-menopausal or glucorticoid-induced OP, bone erosions of rheumatoid arthritis, and bone disease associated with malignant and non-malignant conditions [56]. A reduced OPG/RANKL ratio has been also found in subchondral OBs [57] and chondrocytes of OA patients $[58,59]$. Moreover, celecoxib increased the OPG/RANKL ratio in human OA cartilage [59]. Interestingly, a decrease in this ratio in serum of $\mathrm{OA}$ patients has been related to cartilage damage [60]. Thus, the OPG/RANKL ratio plays a significant role in $\mathrm{OP}$ and likely in OA.

The significant correlations between bone structural, remodelling and molecular parameters in our animal model indicate that alterations in systemic and local remodelling lead to changes at the structural and biomechanical level at subchondral bone in OP, OA and particularly in OPOA group. All these changes contribute to impairment in subchondral bone quality, and make this organ not able to receive and properly distribute loads from and/or to the articular cartilage. Moreover, the significant correlation between subchondral microstructure impairment and cartilage damage denotes that changes at subchondral bone aggravate cartilage damage. Although discrepancies have been raised about subchondral changes originated by the simultaneous presence of $\mathrm{OP}$ and $\mathrm{OA}$ in same individuals, our findings support a synergism of both diseases, particularly when early OA stage is assessed. In addition, it is possible that in post-traumatic OA, trabecular microarchitecture changes are different than in primary OA. In fact, a potential osteopenia by disuse due to decreased load bearing has been proposed during the early post-operative period in the canine ACL-deficient knee [61].

\section{Conclusions}

Thus, although obvious differences exist between idiopathic OA in humans and our rabbit mechanical model of the disease, the results of this work strengthen the role of the subchondral bone as a key player in the puzzle of OA development [12,31,42-45]. Also, they support our previous research [11] by demonstrating that microstructure impairment at subchondral bone associated with an increased remodelling increases cartilage damage in OA rabbits with previous OP. Precisely, in light of these studies, our current findings suggest that an increased subchondral bone resorption may account for the aggravation of cartilage damage when early OA and $\mathrm{OP}$ coexist simultaneously in the same individuals.

\section{Abbreviations}

2D: two-dimensional; ACL: anterior cruciate ligament; ALP: alkaline hosphatise; AU: arbitrary units; B.Ar: bone area; B.Ar/T.Ar\%: bone area fraction; EDTA: ethylenediaminetetraacetic acid; FD: fractal dimension; GAPDH: Glyceraldehyde 3-phosphate dehydrogenase; I: moment of inertia; Ip: polar moment of inertia; m: mass; MMP9: metalloproteinase-9; OPG: osteoprotegerin; PBS: phosphate buffer saline; PVDF: polyvinylidene difluoride; r: perpendicular distance; RANK: receptor activator of nuclear factor- $\kappa$ B; RANKL: receptor activator of nuclear factor- $\kappa$ B ligand; SDS: sodium dodecyl sulphate; SEM: standard error of the mean; SPSS: statistical package for the social sciences; Tb.N: trabecular number; Tb.Sp: trabecular separation; Tb.Th: trabecular thickness; TRAP: tartrate-resistant alkaline phosphastase; $\mu C T$ : micro-computed tomography

\section{Acknowledgements}

This study was supported by research grants from Fondo de Investigacion Sanitaria (FIS) (CP03/0011 and PI06/0032), the Spanish Ministry of Science and Innovation (SAF2006/2704), Fundacion Mutua Madrileña, and Fundacion Mapfre.

\section{Author details}

Bone and Joint Research Unit, Service of Rheumatology. Fundación Jiménez Díaz, Universidad Autónoma. Avda. Reyes Católicos, 2. 28040 Madrid, Spain. ${ }^{2}$ Department of Rheumatology. Hospital de la Princesa, Universidad Autónoma. Calle de Diego de León, 62. 28006 Madrid, Spain. ${ }^{3}$ Trabeculae S.L. Parque Tecnológico de Galicia. 32900 San Cibrao das Viñas, Ourense, Spain. ${ }^{4}$ Department of Orthopaedic Surgery. Fundación Jiménez Díaz, Universidad Autónoma. Avda. Reyes Católicos, 2. 28040 Madrid, Spain.

\section{Authors' contributions}

$\mathrm{RL}$ and $\mathrm{GH}-\mathrm{B}$ designed and conceived of the study. $\mathrm{MB}, \mathrm{LL}, \mathrm{JRC}$ and $\mathrm{DP}$ acquired the data. $M B, L L, J A R-B, S C, R L$ and $G H-B$ analysed and interpreted the data. MB, LL, JA-RB, SC and GH-B drafted the manuscript. All authors revised the manuscript critically for important intellectual content, and all authors approved the final version to be published. GH-B had full access to all of the data in the study and takes responsibility for the integrity of the data and the accuracy of the data analysis.

\section{Competing interests}

The authors declare that they have no competing interests.

Received: 24 April 2010 Revised: 9 June 2010 Accepted: 2 August 2010 Published: 2 August 2010

\section{References}

1. Herrero-Beaumont G, Roman-Blas JA, Castañeda S, Jimenez SA: Primary osteoarthritis: three subsets with etiological, clinical and therapeutic characteristics. Semin Arthritis Rheum 2009, 39:71-80.

2. Sellam J, Herrero-Beaumont G, Berenbaum F: Osteoarthritis: pathogenesis, clinical aspects and diagnosis. Eular Compedium on Rheumatic Diseases 
London, UK: BMJ GroupBijlsma JWJ, Burmester GR, daSilva JAP, Faarvang KL, Hachulla E, Mariette X 2009, 444-463.

3. $\mathrm{NIH}$ Consensus Development Panel on Osteoporosis Prevention Diagnosis and Therapy: Osteoporosis prevention, diagnosis, and therapy. JAMA 2001, 285:785-795

4. Dequeker J, Aerssens J, Luyten FP: Osteoarthritis and osteoporosis: clinical and research evidence of inverse relationship. Aging Clin Exp Res 2003, 15:426-439.

5. Stewart A, Black AJ: Bone mineral density in osteoarthritis. Curr Opin Rheumatol 2000, 12:464-467.

6. Cooper C, Cook PL, Osmond C, Fisher L, Cawley Ml: Osteoarthritis of the hip and osteoporosis of the proximal femur. Ann Rheum Dis 1991, 50:540-542.

7. Zhang Y, Hannan MT, Chaisson CE, McAlindon TE, Evans SR, Aliabadi P, Levy D, Felson DT: Bone mineral density and risk of incident and progressive radiographic knee osteoarthritis in women: the Framingham Study. J Rheumatol 2000, 27:1032-1037.

8. Masud T, Langley S, Wiltshire P, Doyle DV, Spector TD: Effect of spinal osteophytosis on bone mineral density measurements in vertebral osteoporosis. BMJ 1993, 307:172-173.

9. Haddaway MJ, Davie MW, McCall IW: Bone mineral density in healthy normal women and reproducibility of measurements in spine and hip using dual-energy X-ray absorptiometry. Br J Radiol 1992, 65:213-217.

10. Ma HL, Blanchet TJ, Peluso D, Hopkins B, Morris EA, Glasson SS: Osteoarthritis severity is sex dependent in a surgical mouse model. Osteoarthritis Cartilage 2007, 15:695-700.

11. Calvo E, Castañeda S, Largo R, Fernández-Valle ME, Rodríguez-Salvanés F, Herrero-Beaumont G: Osteoporosis increases the severity of cartilage damage in an experimental model of osteoarthritis in rabbits. Osteoarthritis Cartilage 2007, 15:69-77.

12. Radin EL, Rose RM: Role of subchondral bone in the initiation and progression of cartilage damage. Clin Orthop Relat Res 1986, 213:34-40.

13. Burr DB: The importance of subchondral bone in osteoarthrosis. Curr Opin Rheumatol 1998, 10:256-262.

14. Buckland-Wright C: Subchondral bone changes in hand and knee osteoarthritis detected by radiography. Osteoarthritis Cartilage 2004 12(Suppl A):S10-9.

15. Chappard C, Peyrin F, Bonnassie A, Lemineur G, Brunet-Imbault B, Lespessailles E, Benhamou CL: Subchondral bone micro-architectural alterations in osteoarthritis: a synchrotron micro-computed tomography study. Osteoarthritis Cartilage 2006, 14:215-223.

16. Bobinac D, Spanjol J, Zoricic S, Maric I: Changes in articular cartilage and subchondral bone histomorphometry in osteoarthritic knee joints in humans. Bone 2003, 32:284-290.

17. Messent EA, Ward RJ, Tonkin CJ, Buckland-Wright C: Cancellous bone differences between knees with early, definite and advanced joint space loss; a comparative quantitative macroradiographic study. Osteoarthritis Cartilage 2005, 13:39-47.

18. Hart DJ, Cronin C, Daniels M, Worthy T, Doyle DV, Spector TD: The relationship of bone density and fracture to incident and progressive radiographic osteoarthritis of the knee: The Chingford study. Arthritis Rheum 2002, 46:92-99.

19. Pastoureau P, Leduc S, Chomel A, De Ceuninck F: Quantitative assessment of articular cartilage and subchondral bone histology in the meniscectomized guinea pig model of osteoarthritis. Osteoarthritis Cartilage 2003, 11:412-413.

20. Ledingham J, Dawson S, Preston B, Milligan G, Doherty M: Radiographic patterns and associations of osteoarthritis of the hip. Ann Rheum Dis 1992, 51:1111-1116.

21. Bierma-Zeinstra SM, Koes BW: Risk factors and prognostic factors of hip and knee osteoarthritis. Nat Clin Pract Rheumatol 2007, 3:78-85.

22. Conrozier T, Merle-Vincent F, Mathieu P, Richard M, Favret $H$, Piperno M, Caton J, Vignon E: Epidemiological, clinical and radiological differences between atrophic and hypertrophic patterns of hip osteoarthritis: a case-control study. Clin Exp Rheumatol 2004, 22:403-408.

23. Roemer FW, Neogi T, Nevitt MC, Felson DT, Zhu Y, Zhang Y, Lynch JA, Javaid MK, Crema MD, Torner J, Lewis CE, Guermazi A: Subchondral bone marrow lesions are highly associated with, and predict subchondral bone attrition longitudinally: the MOST study. Osteoarthritis Cartilage 2010, 18:47-53.
24. Conrozier $T$, Ferrand $F$, Poole $A R$, Verret $C$, Mathieu $P$, lonescu $M$, Vincent $F$, Piperno M, Spiegel A, Vignon E: Differences in biomarkers of type II collagen in atrophic and hypertrophic osteoarthritis of the hip: implications for the differing pathobiologies. Osteoarthritis Cartilage 2007, 15:462-467.

25. Bettica P, Cline G, Hart DJ, Meyer J, Spector TD: Evidence for increased bone resorption in patients with progressive knee osteoarthritis: longitudinal results from the Chingford study. Arthritis Rheum 2002, 46:3178-3184.

26. Bailey AJ, Mansell JP, Sims TJ, Banse X: Biochemical and mechanical properties of subchondral bone in osteoarthritis. Biorheology 2004, 41:349-358.

27. Neogi T, Felson D, Niu J, Lynch J, Nevitt M, Guermazi A, Roemer F, Lewis CE, Wallace B, Zhang Y: Cartilage loss occurs in the same subregions as subchondral bone attrition: a within-knee subregionmatched approach from the multicenter osteoarthritis study. Arthritis Rheum 2009, 61:1539-1544.

28. Botter SM, van Osch GJ, Waarsing JH, Day JS, Verhaar JA, Pols HA, van Leeuwen JP, Weinans H: Quantification of subchondral bone changes in a murine osteoarthritis model using micro-CT. Biorheology 2006 43:379-388.

29. Lavigne P, Benderdour M, Lajeunesse D, Reboul P, Shi Q, Pelletier JP, Martel-Pelletier J, Fernandes JC: Subchondral and trabecular bone metabolism regulation in canine experimental knee osteoarthritis. Osteoarthritis Cartilage 2005, 13:310-317.

30. Calvo E, Palacios I, Delgado E, Ruiz-Cabello J, Hernández P, Sánchez Pernaute $\mathrm{O}$, Egido J, Herrero-Beaumont G: High-resolution MRI detects cartilage swelling at the early stages of experimental osteoarthritis. Osteoarthritis Cartilage 2001, 9:463-472.

31. Karsdal MA, Leeming DJ, Dam EB, Henriksen K, Alexandersen $P$, Pastoureau P, Altman RD, Christiansen C: Should subchondral bone turnover be targeted when treating osteoarthritis? Osteoarthritis Cartilage 2008, 16:638-646.

32. Kwan Tat S, Pelletier JP, Lajeunesse D, Fahmi H, Lavigne M, MartelPelletier J: The differential expression of osteoprotegerin (OPG) and receptor activator of nuclear factor kappaB ligand (RANKL) in human osteoarthritic subchondral bone osteoblasts is an indicator of the metabolic state of these disease cells. Clin Exp Rheumatol 2008, 26:295-304.

33. Sakao K, Takahashi KA, Mazda O, Arai $Y$, Tonomura H, Inoue A, Saito M, Fujioka M, Takamiya $H$, Imanishi J, Kubo T: Enhanced expression of interleukin-6, matrix metalloproteinase-13, and receptor activator of NFkappaB ligand in cells derived from osteoarthritic subchondral bone. $J$ Orthop Sci 2008, 13:202-210.

34. Takayanagi $\mathrm{H}$, Kim S, Taniguchi T: Signaling crosstalk between RANKL and interferons in osteoclast differentiation. Arthritis Res 2002, 4(Suppl 3): S227-232.

35. Pothuaud L, Benhamou CL, Porion P, Lespessailles E, Harba R, Levitz P: Fractal dimension of trabecular bone projection texture is related to three-dimensional microarchitecture. J Bone Miner Res 2000, 15:691-699.

36. Liu L, Maruno R, Mashimo T, Sanka K, Higuchi T, Hayashi K, Shirasaki Y, Mukai N, Saitoh S, Tokuyama K: Effects of physical training on cortical bone at midtibia assesses by peripheral QCT. J Appl Physiol 2003, 95:219-224.

37. Mankin HJ, Dorfman H, Lippiello L, Zarins A: Biochemical and metabolic abnormalities in articular cartilage from osteo-arthritic human hips. II. Correlation of morphology with biochemical and metabolic data. J Bone Joint Surg Am 1971, 53:523-537.

38. Calvo E, Palacios I, Delgado E, Sanchez-Pernaute O, Largo R, Egido J, Herrero-Beaumont G: Histopathological correlation of cartilage swelling detected by magnetic resonance imaging in early experimental osteoarthritis. Osteoarthritis Cartilage 2004, 12:878-886.

39. Cake MA, Appleyard RC, Read RA, Smith MM, Murrell GA, Ghosh P: Ovariectomy alters the structural and biomechanical properties of ovine femoro-tibial articular cartilage and increases cartilage iNOS Osteoarthritis Cartilage 2005, 13:1066-1075.

40. Claassen H, Hornberger F, Scholz-Ahrens K, Schünke M, Schrezenmeir J, Kurz B: The effect of estrogens and dietary calcium deficiency on the extracellular matrix of articular cartilage in Göttingen miniature pigs. Ann Anat 2002, 184:141-148. 
41. Oestergaard S, Sondergaard BC, Hoegh-Andersen P, Henriksen K, Qvist P, Christiansen C, Tankó LB, Karsdal MA: Effects of ovariectomy and estrogen therapy on type II collagen degradation and structural integrity of articular cartilage in rats: implications of the time of initiation. Arthritis Rheum 2006, 54:2441-2451.

42. Sniekers $Y H$, Intema F, Lafeber FP, van Osch GJ, van Leeuwen JP, Weinans $\mathrm{H}$, Mastbergen SC: A role for subchondral bone changes in the process of osteoarthritis; a micro-CT study of two canine models. BMC Musculoskelet Disord 2008, 9:20.

43. Kadri A, Ea HK, Bazille C, Hannouche D, Lioté F, Cohen-Solal ME: Osteoprotegerin inhibits cartilage degradation through an effect on trabecular bone in murine experimental osteoarthritis. Arthritis Rheum 2008, 58:2379-2386.

44. Hayami T, Pickarski M, Zhuo Y, Wesolowski GA, Rodan GA, Duongle T: Characterization of articular cartilage and subchondral bone changes in the rat anterior cruciate ligament hosphatise and meniscectomized models of osteoarthritis. Bone 2006, 38:234-243.

45. Hayami T, Pickarski M, Wesolowski GA, McLane J, Bone A, Destefano J, Rodan GA, Duongle T: The role of subchondral bone hosphatise in osteoarthritis: reduction of cartilage degeneration and prevention of osteophyte formation by alendronate in the rat anterior cruciate ligament hosphatise model. Arthritis Rheum 2004, 50:1193-1206.

46. Yi WJ, Heo MS, Lee SS, Choi SC, Huh KH: Comparison of trabecular bone anisotropies based on fractal dimensions and mean intercept length determined by principal axes of inertia. Med Biol Eng Comput 2007, 45:357-364.

47. Fazzalari NL, Darracott J, Vernon-Roberts B: Histomorphometric changes in the trabecular structure of a selected stress region in the femur in patients with osteoarthritis and fracture of the femoral neck. Bone 1985, 6:125-133.

48. Messent EA, Ward RJ, Tonkin CJ, Buckland-Wright C: Cancellous bone differences between knees with early, definite and advanced joint space loss; a comparative quantitative macroradiographic study. Osteoarthritis Cartilage 2005, 13:39-47.

49. An HS, Schwab JP, Skrade D: Biological Tissues and functional biomechanics. Synopsis of Orthopaedics New York, NY, USA: Thieme Medical Publishers IncAn HS 1992, 491-492.

50. Mukherjee M, Das AS, Mitra S, Mitra C: Prevention of bone loss by oil extract of garlic (Allium sativum Linn.) in an ovariectomized rat model of osteoporosis. Phytother Res 2004, 18:389-394.

51. Mansell JP, Tarlton JF, Bailey AJ: Biochemical evidence for altered subchondral bone collagen metabolism in osteoarthritis of the hip. $\mathrm{Br} J$ Rheumatol 1997, 36:16-19.

52. Shibakawa A, Yudoh K, Masuko-Hongo K, Kato T, Nishioka K, Nakamura H: The role of subchondral bone resorption pits in osteoarthritis: MMP production by cells derived from bone marrow. Osteoarthritis Cartilage 2005, 13:679-687.

53. Clements DN, Fitzpatrick N, Carter SD, Day PJ: Cartilage gene expression correlates with radiographic severity of canine elbow osteoarthritis. Vet $J$ 2009, 179:211-218.

54. Hulejová H, Baresová V, Klézl Z, Polanská M, Adam M, Senolt L: Increased level of cytokines and matrix metalloproteinases in osteoarthritic subchondral bone. Cytokine 2007, 38:151-156.

55. Leibbrandt A, Penninger JM: RANK/RANKL: regulators of immune responses and bone physiology. Ann N Y Acad Sci 2008, 1143:123-150.

56. Hofbauer LC, Schoppet M: Clinical implications of the osteoprotegerin/ RANKL/RANK system for bone and vascular diseases. JAMA 2004, 292:490-495.

57. Tat SK, Pelletier JP, Lajeunesse D, Fahmi H, Duval N, Martel-Pelletier J: Differential modulation of RANKL isoforms by human osteoarthritic subchondral bone osteoblasts: influence of osteotropic factors. Bone 2008, 43:284-291.

58. Moreno-Rubio J, Herrero-Beaumont G, Tardío L, Alvarez-Soria MA, Largo R: Nonsteroidal antiinflammatory drugs and prostaglandin $\mathrm{E}(2)$ modulate the synthesis of osteoprotegerin and RANKL in the cartilage of patients with severe knee osteoarthritis. Arthritis Rheum 2010, 62:478-488.

59. Kwan Tat S, Amiable N, Pelletier JP, Boileau C, Lajeunesse D, Duval N, Martel-Pelletier J: Modulation of OPG, RANK and RANKL by human chondrocytes and their implication during osteoarthritis. Rheumatology Oxford 2009, 48:1482-1490.
60. Pilichou A, Papassotiriou I, Michalakakou K, Fessatou S, Fandridis E, Papachristou G, Terpos E: High levels of synovial fluid osteoprotegerin (OPG) and increased serum ratio of receptor activator of nuclear factorkappa B ligand (RANKL) to OPG correlate with disease severity in patients with primary knee osteoarthritis. Clin Biochem 2008, 41:746-749.

61. Boyd SK, Muller R, Matyas JR, Wohl GR, Zernicke RF: Early morphometric and anisotropic change in periarticular cancellous bone in a model of experimental knee osteoarthritis quantified using microcomputed tomography. Clin Biomech (Bristol, Avon) 2000, 15:624-631.

doi:10.1186/ar3103

Cite this article as: Bellido et al:: Subchondral bone microstructural damage by increased remodelling aggravates experimental osteoarthritis preceded by osteoporosis. Arthritis Research \& Therapy 2010 12:R152.

\section{Submit your next manuscript to BioMed Central and take full advantage of:}

- Convenient online submission

- Thorough peer review

- No space constraints or color figure charges

- Immediate publication on acceptance

- Inclusion in PubMed, CAS, Scopus and Google Scholar

- Research which is freely available for redistribution

Submit your manuscript at www.biomedcentral.com/submit
C Biomed Central 